\title{
Savoir culturel et langue en danger : l'exemple du rituel de l'igname chez les Kwoma
}

Cultural knowledge and endangered language: The case of the Kwoma yam ritual

\section{Renée Lambert-Brétière}

\section{CpenEdition}

\section{Journals}

Édition électronique

URL : http://journals.openedition.org/jso/8624

DOI : $10.4000 /$ jso.8624

ISSN : $1760-7256$

Éditeur

Société des océanistes

Édition imprimée

Date de publication : 15 juillet 2018

Pagination : 73-83

ISBN : 978-2-85430-135-9

ISSN : 0300-953x

Référence électronique

Renée Lambert-Brétière, « Savoir culturel et langue en danger : l'exemple du rituel de l'igname chez les Kwoma », Journal de la Société des Océanistes [En ligne], 146 | 2018, mis en ligne le 15 juillet 2020, consulté le 25 juillet 2020. URL : http://journals.openedition.org/jso/8624 ; DOI : https://doi.org/ $10.4000 / j s 0.8624$

(c) Tous droits réservés 


\title{
Savoir culturel et langue en danger : l'exemple du rituel de l'igname chez les Kwoma
}

par

\author{
Renée LAMBERT-BRÉTIÈRE*
}

\section{RÉSUMÉ}

L'objectif de cet article est dillustrer la relation intrinsèque existant chez les Kwoma entre le rituel de l'igname et la connaissance linguistique associée aux chants invoqués lors de sa pratique. Le rituel autour de l'igname est constitué de trois cérémonies distinctes, Yena, Mija et Nowkwi, et inclut des chants et des danses en l'honneur de ces esprits. Les chants rituels sont une source de savoir pour l'histoire mais aussi sur les valeurs et les normes socioculturelles à l'auvre dans la communauté. Après une présentation de la structure sociale de la société kwoma, un examen du rituel de ligname puis une analyse de deux chants rituels, sawo howkwa et magwiy howkwa, on montrera que la sauvegarde du patrimoine culturel kwoma ne peut se faire sans la sauvegarde du patrimoine culturel immatériel.

MoTs-ClÉs : langue, chants, rituel de l'igname, patrimoine culturel immatériel, Kwoma

La mondialisation a accéléré le déclin de plusieurs langues et cultures. Des quelques sept mille langues étant encore parlées aujourd'hui, plus de la moitié est appelée à disparaître d'ici la fin de ce siècle, entraînant avec elle quantités de savoirs traditionnels et de matériaux culturels (Crystal, 2000). Lorsque les communautés perdent leur langue, leurs traditions culturelles orales, telles que les chants, les mythes et la poésie qui sont plus difficilement transférables d'une langue à l'autre, disparaissent (Le Tollec, 2013). Devant cette menace, l'UNESCO a réagi en mettant sur pied la

\section{ABSTRACT}

The purpose of this article is to illustrate the intrinsic relationship among the Kwoma between the practice of the yam ritual and the linguistic knowledge associated with the songs and formulas invoked during its performance. The yam ritual is composed of three distinct ceremonies, Yena, Mija and Nowkwi, and includes songs and dances to honor these spirits. Ritual songs are a source of knowledge about history and the socio-cultural values and standards expected in this community. After an overview of the social structure of the Kwoma society, a presentation of the yam ritual, and an analysis of two ritual songs - sawo howkwa and magwiy howkwa -, this article demonstrates that the preservation of the Kwoma cultural heritage cannot be done at the expense of its intangible cultural heritage.

KeYwords: Language, songs, yam ritual, intangible cultural heritage, Kwoma

Convention pour la sauvegarde du patrimoine immatériel, entrée en vigueur en 2006. Les objectifs de cette convention sont notamment la sauvegarde, le respect et la sensibilisation à l'importance du patrimoine culturel immatériel. Ce patrimoine est formé :

" des expressions, des valeurs, des coutumes, des langues, de la tradition orale, des modes de vie traditionnels, de la créativité, de l'adaptabilité et, en définitive, de tout ce qui fait le caractère distinctif d'un peuple. " (Grattan, 2006)

\footnotetext{
* Professeure adjointe de linguistique et de français, Département de langues modernes, linguistique et communication interculturelle, Université du Maryland (Comté de Baltimore), rlambert@umbc.ed
} 


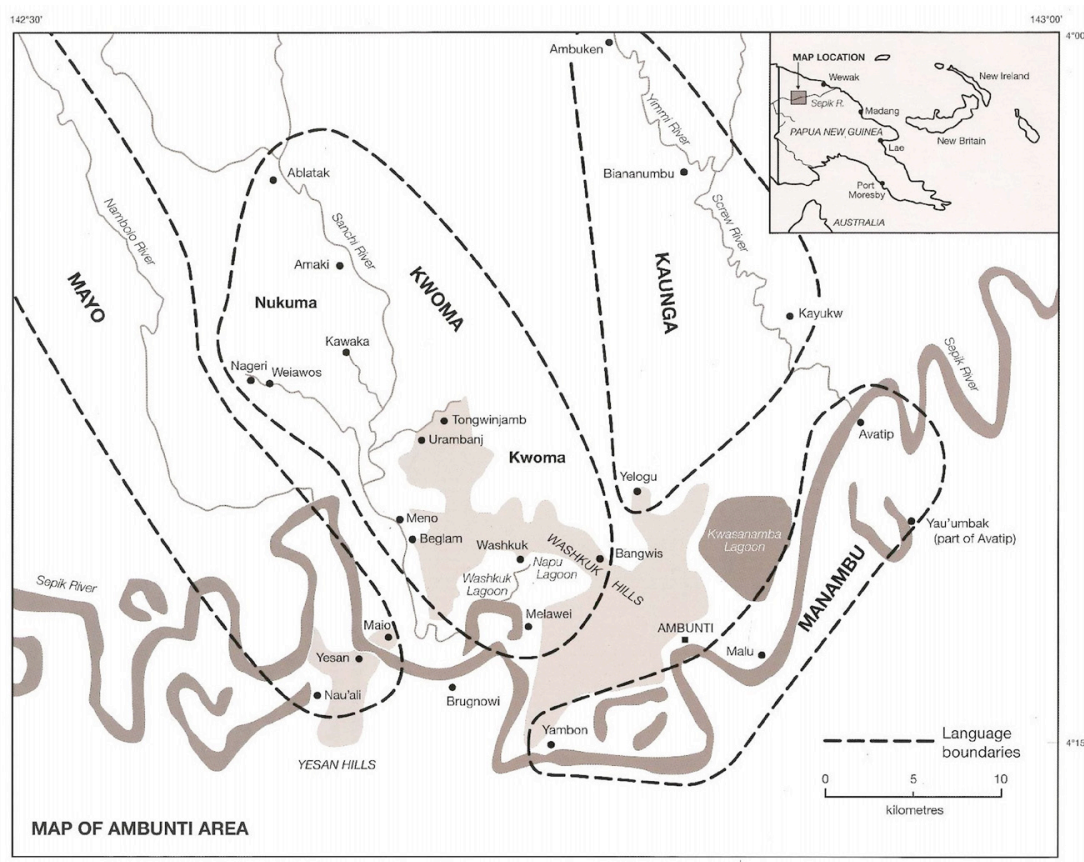

Carte 1. - Territoire Kwoma (C Dr. Ross Bowden, avec nos remerciements)

boldt (1859: 54) l'idée que la langue " contient l'ensemble des idées et la manière de voir" d'un peuple, ou encore chez Boas (1911) la conception que «la langue est un fait de culture, et [que] la culture se dit dans la langue " (cité dans Bornand et Leguy, 2013: 2). Ainsi, la diversité linguistique contient une extraordinaire richesse dans la quantité d'informations encodée dans chaque langue, qu'il s'agisse de connaissances sur les pratiques culturelles, la nourriture, l'environnement, la santé et les modes de vie. Chaque langue représente un moyen de communica-

La langue constitue l'un des éléments fondamentaux de la culture et imprègne tous les aspects de l'expérience humaine. En plus de faire partie du patrimoine culturel immatériel d'un peuple, la langue constitue une réflexion complète et complexe de celui-ci (Arpin, 2001). Elle est composée de règles socialement partagées qui permettent aux locuteurs de cette langue de savoir ce que les mots signifient, de créer de nouveaux mots, de mettre les mots ensemble et de choisir la formulation appropriée au contexte. Les hommes utilisent le langage pour exprimer leurs pensées et leurs émotions, pour donner un sens au monde qui les entoure et pour transmettre la connaissance. Chaque langue est :

« un instrument de communication selon lequel l'expérience humaine s'analyse, différemment dans chaque communauté. » (Martinet, 2005 : 20)

Les connaissances linguistiques ne sont jamais neutres; elles constituent des représentations du monde constamment utilisées pour la construction d'affinités et de différenciations culturelles (Duranti, 1997). Selon Maynard Smith et Szathmáry (1995), le concept même d'évolution culturelle est dépendant de la capacité des humains à utiliser la langue pour maintenir leur cohésion sociale. Cette idée qu'il existe une corrélation entre la forme et le contenu d'une langue et les croyances, les valeurs et les besoins culturels de ses locuteurs n'est pas nouvelle. On trouve déjà chez von Hum- tion distinct et illustre bien la créativité de l'esprit humain. L'étude des langues en voie de disparition devient ainsi un facteur prépondérant dans la sauvegarde du patrimoine culturel immatériel.

L'extrême diversité linguistique et culturelle en Océanie s'illustre entre autres par les quelques neuf cents langues dites papoues ${ }^{1}$. Il est difficile de mesurer le taux de disparition de ces langues, puisque moins de $10 \%$ des langues papoues ont été documentés de façon détaillée (voir Foley, 1986, 2000 ; Aikhenvald et Stebbins, 2007). L'étude présentée dans cet article s'inscrit dans un projet de recherche plus large de sauvegarde du patrimoine culturel immatériel des Kwoma, un peuple habitant la province Est-Sepik en Papouasie Nouvelle-Guinée. La langue kwoma est l'une des quelques quatrevingt-dix langues faisant partie de la branche Sepik-Ramu des langues papoues (Simons et Fennig, 2017). Selon les données du recensement de 2011, il y a environ quatre milles locuteurs du kwoma, dispersés dans sept villages localisés dans les monts Washkuk au nord d'Ambunti dans le district Ambunti-Dreikikir.

Le kwoma est parlé par un nombre relativement élevé de locuteurs. Dans la vie quotidienne, les femmes et les enfants parlent kwoma, alors que les hommes utilisent plus fréquemment le tok pisin. Les hommes utilisent le kwoma dans des situations plus traditionnelles (chants, cérémonies, réunions dans les maisons des esprits). Seuls quelques locuteurs, généralement les hommes les plus éduqués, parlent l'anglais. Le tok pisin est la langue domi-

1. Il est à noter qu'il n'y a aucun lien génétique démontré avec certitude entre les diverses familles composant le phylum de langues papoues. Pour cette raison, les langues papoues sont parfois appelées « langues non austronésiennes " par opposition à l'autre groupe de langues dominant de Papouasie Nouvelle-Guinée (Foley, 1986, 1998, 2000). 
nante utilisée dans l'éducation publique, les transactions officielles et les cérémonies se déroulant à l'église. Ainsi, le tok pisin remplace progressivement le kwoma, particulièrement dans les villages situés près d'Ambunti, la capitale multilingue du district. La situation culturelle reflète la situation linguistique. La colonisation, le christianisme et l'abolition de la chasse aux têtes ont bouleversé les pratiques sociales et culturelles. Plusieurs cérémonies et rituels, comme par exemple les rituels de la puberté ou la cérémonie de la plantation de l'igname documentés par l'anthropologue John W. M. Whiting dans les années 1940, ont déjà disparu (Whiting, 1941). La transformation de la vie rituelle des Kwoma a laissé derrière elle peu d'hommes pouvant réaliser une grande partie des rituels et cérémonies jadis courants dans la vie des Kwoma. Mais, il existe encore, par exemple, dans certains villages, une cour de justice traditionnelle. Le rôle premier de cette justice est, entre autres, de maintenir la paix et l'harmonie dans le village. Les aînés de chaque clan se réunissent et prennent des décisions en fonction du bien de la communauté et non des intérêts individuels. Les jugements sont rendus dans une langue kwoma traditionnelle qu'ils sont les seuls à maîtriser. L'apparition d'une police nationale diminue la fréquence de ces tribunaux et, de fait, la transmission de ce type de discours oratoire en langue traditionnelle. Il existe en effet plusieurs niveaux de langue, un parler courant en constante évolution et un plus figé utilisé lors de cérémonies traditionnelles que les jeunes Kwoma ont du mal à comprendre.

Les données présentées dans cet article proviennent d'observations ethnographiques de terrain faites lors de trois séjours dans le village de Tongwinjamb ${ }^{2}$, entre 2006 à 2008 et cumulant un total de neuf mois d'enquêtes. À travers mes séjours chez les Kwoma, j'ai appris le tok pisin et les bases de communication en tok ples, "langue native", ce qui m’a permis de mieux m'intégrer dans la communauté et de développer des relations durables avec de nombreux villageois. Ma place dans le système de parenté fut négociée lors de mon premier séjour chez les Kwoma en août 2006. Il fut convenu que j'aurais le même statut que ma consultante principale et, qu'en conséquence, je devenais sa jeune sœur (kumwey). Lors de mes voyages, j'ai été accompagnée par mon mari qui s'est vu attribuer un statut identique (kumwey) $)^{3}$. Pour mon dernier voyage, en 2008, notre fils, âgé de dix-huit mois, est venu avec nous et a lui aussi intégré le système de parenté (ruwey). La présence de ma famille s'est avérée très importante, puisque que, comme je l'expliquerai ci-dessous, mon mari a pu accéder à du matériel culturel interdit aux femmes.

Dans cette société à tradition orale, la transmission du savoir repose sur la langue. L'objectif de cet article est de démontrer la relation intrinsèque existant entre la pratique d'un rituel, celui de l'igname, et la connaissance des chants et des formules prononcés lors de celui-ci. Ce rituel, composé de trois cérémonies distinctes Yena, Mija et Nowkwi, l'un des mieux connus des rituels kwoma, est grandement menacé par la perte du savoir linguistique qui en permet la réalisation. Après un survol de la structure sociale de la société, j'aborderai la description du cycle rituel de l'igname en en présentant les traits caractéristiques et en en étudiant deux chants rituels, sawo howkwa et magwiy howkwa. Il ressortira de cette analyse que la préservation de ce rituel est indissociable du processus de sa transmission par la langue. Le rôle du rituel de l'igname dépasse largement celui de sa mise en culture et de sa récolte. Ce rituel fait partie intégrante du processus de continuité : il est au cœur du lien intergénérationnel maintenu grâce à la transmission des traditions d'une génération à l'autre.

\section{Les Kwoma}

Les Kwoma habitent dans le district d'Ambunti-Dreikikir dans la vallée du Sepik en Papouasie Nouvelle-Guinée (voir Carte 1). La population est divisée en deux sous-groupes de communautés. L'un est situé au pied des collines Washkuk et ses membres s'identifient comme Kwoma, c'està-dire des " hommes des collines ". Le second est situé au nord et à l'ouest des monts Washkuk et ses membres s'identifient comme Nukuma, c'està-dire des " hommes de l'eau " ${ }^{4}$. Le terme kwoma peut être employé en référence à l'ensemble du peuple kwoma et regroupe ainsi les Kwoma et les Nukuma (voir par exemple Bowden, 1997). Dans cet article, j'utilise le terme kwoma en référence au sous-groupe kwoma, excluant de facto les Nukuma5. Jusque dans les années 1940, tous les Kwoma habitaient en haut des collines Washkuk.

2. Dans la documentation officielle du gouvernement de Papouasie Nouvelle-Guinée, on trouve «Tangujamb ». Au village, il est écrit «Tongunjamb » sur la façade du centre médical et « Tongujamb » sur celle de l'école primaire. Dans son dictionnaire, Bowden (1997) écrit "Tongwinjamb ». C'est cette dernière orthographe que j’ai décidé d'utiliser dans cet article.

3. En langue kwoma, la même forme est adressée à la jeune sœur et à son époux, alors qu'en tok pisin, des termes distincts sont utilisés (liklik susa " petite sœur ", tambu «beau-frère »). Voir Bowden (1983b) pour une présentation détaillée du système de parenté chez les Kwoma.

4. Le terme kwoma résulte de la combinaison de kwo " mont» et de ma " homme ». Le terme nukuma est formé de nuku " embouchure " et de ma « homme».

5. Cette décision est motivée par le fait que la population Kwoma avec laquelle nous avons travaillé se distingue clairement des Nukuma qu'elle désigne comme étant « les autres ». Cependant, il est probable que plusieurs faits discutés dans cet article s'appliquent également au contexte culturel des Nukuma. 
Ils vivaient dans de petits hameaux isolés sur les divers pans d'une même colline, comme le signale Whiting dans son étude ethnographique (1941). Suite à la suppression des guerres intertribales par le gouvernement colonial australien après la Deuxième Guerre mondiale, les villages kwoma furent installés au bas des collines afin de faciliter l'accès à l'eau. Cette opération est à l'origine de la division de certains hameaux en plusieurs villages distincts. Ces villages ont cependant conservé, en gros, la même organisation sociale.

Les Kwoma ont une résidence patrilocale et une descendance patrilinéaire. Ainsi, les Kwoma habitent de grands hameaux, divisés en petits hameaux habités par les membres d'un lignage patrilinéaire avec leurs femmes, les filles de ce lignage quittant le hameau lors de leur mariage. Plusieurs hameaux (de trois à huit) forment un village. Le village (akama) constitue l'unité politique de base. Selon Bowden (2006), le peuple Kwoma se subdivise en quatre tribus (magwiy) : les Hogwama, les Kowariyasi, les Tokogwiyishebi et les Wurumbaj. À travers tout le territoire kwoma, les tribus se regroupent en fonction de leurs relations patrilinéaires, en une série de clans exogames (également appelés magwiy). Chaque tribu est divisée en une vingtaine de clans qui peuvent se répartir entre plusieurs villages. Ainsi, la tribu Hogwama comprend dix-huit clans qui habitent dans trois villages distincts, Bangus, Washkuk et Melawei. Mais les clans de la tribu Tokogwiyishebi habitent tous le village de Tongwinjamb. Ce village est le plus important par sa taille et son nombre d'habitants. Selon le recensement de 2011, la moitié de la population kwoma habitait le village de Tongwinjamb, soit mille quatre cent soixante-cinq personnes, dont sept cent dix hommes et sept cent cinquante-cing femmes. En conséquence, depuis les années 1970, le village de Tongwinjamb s'est subdivisé en quatre hameaux comprenant les membres de mêmes clans. Ces hameaux ne sont pas reconnus officiellement comme des villages différents; les habitants les nomment Tonga-1, Tonga- 2 , Tonga- 3 et Tonga- 4 .

En théorie, les membres d'un même clan descendent d'un ancêtre commun masculin selon des liens agnats (yimana " descendant "). Certains clans sont reliés par leurs fondateurs à d'autres groupes exogames, mais ces unités patrilinéaires plus larges ne portent pas de nom spécifique et ne forment pas une unité sociopolitique. Chaque clan possède un grand nombre de totems $(s a b o)$, principalement des plantes et des espèces animales (voir tab. 1). Les totems sont classés par genre, mâle ou femelle: les totems mâles (par exemple les différents types de poissons) constituent la majorité des noms d'hommes, alors que les totems femelles (par exemple la plupart des oiseaux incluant le casoar et l'oiseau de paradis) constituent des noms de femmes. Les membres d'un clan ayant le même totem se considèrent parents (nobo yaka kumwey, "parenté totémique classificatoire »). Les unions par le mariage autorisent l'entrée des femmes dans le clan de leur époux, et elles prennent le totem de celui-ci. À ce propos, il arrive d'identifier un homme dans certaines cérémonies en nommant le totem de son père et le totem originel de sa mère.

Tableau 1. - Exemple de clans kwoma et leurs totems (Bowden, $2006: 14$ )

\begin{tabular}{|c|c|}
\hline Clans & Exemples de totems \\
\hline 1. Giley & $\begin{array}{l}\text { gey « lézard », Veranus salvadorii } \\
\text { sagwa « tarentule », Selenocosmia } \\
\text { crassipes }\end{array}$ \\
\hline 2. Hamikwa & $\begin{array}{l}\text { manu « oiseau de paradis », Paradi- } \\
\text { saea raggiana } \\
\text { aponyumay « aigle », Harpyopsis } \\
\text { novaeguineae }\end{array}$ \\
\hline 3. Keyava & $\begin{array}{l}\text { mo « crocodile », Crocodylus novae- } \\
\text { guineae } \\
\text { ya } \text { « soleil » }\end{array}$ \\
\hline 4. Teki & $\begin{array}{l}\text { aw } \ll \text { poterie } » \\
\text { as } a \text { 《hien } », \text { Canis familiaris }\end{array}$ \\
\hline 5. Wanyi & $\begin{array}{l}\text { aposheb « casoar », Casuarius } \\
\text { poyi « sanglier », Sus scrofa }\end{array}$ \\
\hline 6. Wurabaj & $\begin{array}{l}\text { yirimag « opossum », Dactylopsila } \\
\text { trivirgata } \\
\text { mawuj « palmier à bétel », Areca } \\
\text { catechu }\end{array}$ \\
\hline
\end{tabular}

Lors de mes séjours sur le terrain, ma relation avec les gens s'est négociée par mon adoption dans le système de parenté, par l'attribution de mon prénom à un membre du clan ${ }^{6}$ et, surtout, par la promesse de mon retour et de ma participation morale et financière à la vie du clan. Étant donné que ma présence dans la communauté était liée à mes recherches, il a été entendu que j'intègre la tribu Tokogwiyishebi, en particulier le clan Hamikwa ayant pour totem l'oiseau de paradis $(m a n u)$. Ce statut m'a donné un accès à la vie et aux traditions de mon clan. Le plus souvent, les membres du village venaient me raconter des histoires traditionnelles, comme par exemple l'histoire de Tapabisharakwa, l'homme qui avait des ongles coupant, ou encore l'histoire de Valey, un fantôme dont le chant attire les vivants dans le monde des esprits. En d'autres occasions, ils proposaient des narrations procédurales (comment chasser les crocodiles ( $m o$ ), comment attraper des poissons avec un filet de pêche traditionnel (maya), comment tisser un sac de corde traditionnel (kow), etc.). J'ai également assisté à de nom-

6. Il est pratique courante de donner le prénom de la personne nouvellement intégrée au clan à un enfant, scellant la relation filiale. Ainsi, une petite fille de mon clan a été nommée Renée et mon fils a reçu le prénom kwoma Mehi. 
breux événements d'importance ethnolinguistique, par exemple une cérémonie de deuil (aka kepi), un échange de mima ya "prix de la fiancée " et une cour de justice civile traditionnelle (anasa). Plusieurs Kwoma ont partagé avec moi leur inquiétude quant à la préservation de leur langue et de leur culture. Cette préoccupation est également exprimée par de nombreux linguistes et anthropologues (par exemple Harrison, 2007), qui soulignent que la perte des façons de parler conduit à la perte de connaissances sur la culture. Cela est vrai pour les rituels et les célébrations culturelles qui ne peuvent se réaliser sans la langue rituelle, un Kwoma beaucoup plus soutenu et immuable que les jeunes générations ne maitrisent pas. Je me suis concentrée sur le cycle rituel de l'igname pour démontrer ce phénomène.

\section{Le cycle rituel de l'igname}

Comme les autres sociétés de la région, l'économie des Kwoma est basée principalement sur le sagou (noku, Metroxylon sagu), produit du sagoutier, un type de palmier qui foisonne dans les forêts de la région. Cependant, la nourriture la plus prestigieuse n'est pas le sagou, mais bien l'igname (kwow, Dioscorea esculenta; neny, Dioscorea alata).

Le rituel kwoma le plus important est en association avec la culture de l'igname (kwow sukwiya) ${ }^{7}$. L'igname est considérée comme une entité surnaturelle. On ne peut la consommer que lorsque les esprits responsables de sa culture ont été honorés. Ce rituel est exécuté dans les grandes maisons cérémonielles korobo, aussi appelées «maisons des hommes " (en tok pisin, haus boi) ou " maison des esprits " (en tok pisin, haus tambaran). La photo 1 prise lors de mon séjour en 2007 représente une maison cérémonielle située au sommet d'une colline dans le village de Tonga-3. Comme on peut le voir sur cette photographie, les maisons cérémonielles kwoma sont ouvertes aux deux extrémités et sont constituées d'un toit à forte pente recouvert de feuilles de cocotiers et de sagoutier entrelacées. Elles mesurent entre dix et vingt mètres de long. Les plafonds des maisons cérémonielles sont couverts de pétioles de sagoutiers peints mesurant environ cinquante centimètres sur un mètre. Les artistes commencent par appliquer un fond noir puis dessinent les totems et autres représentations culturelles appartenant à leur clan. Toutes les poutres de soutien du toit et de charpente sont faites de troncs d'arbres sculptés et peints dans les

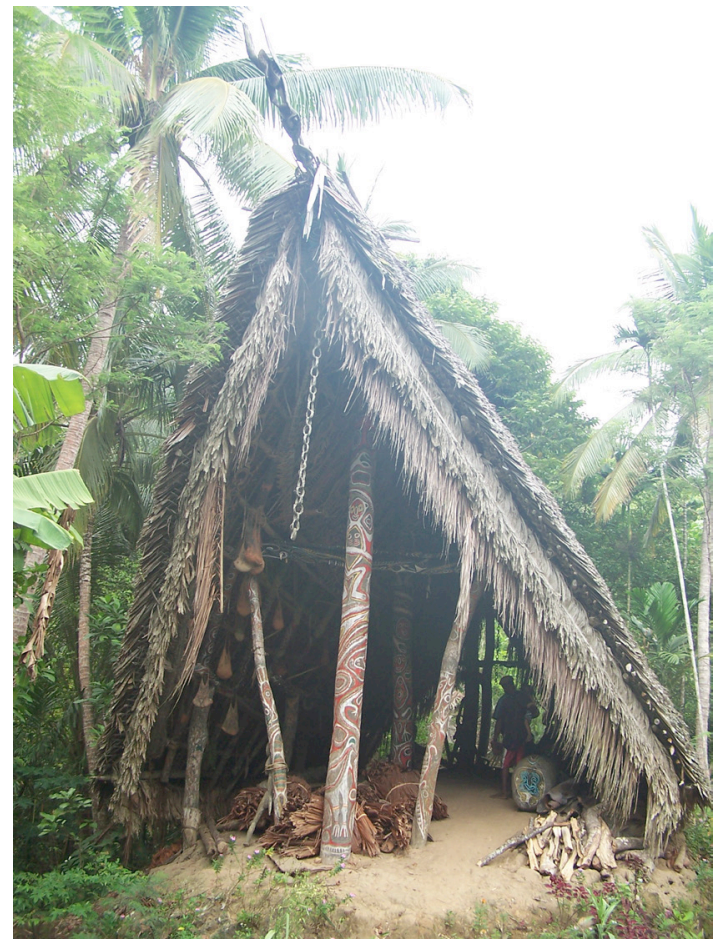

Рното 1. - Korobo "maison cérémonielle" située à Tonga-3, Tongwinjamb, 14 août 2006 (C R. Lambert-Brétière)

mêmes couleurs. Les couleurs traditionnelles sont l'ocre, le brun, le rouge et le blanc ${ }^{8}$.

Les Kwoma n'entretiennent pas les maisons des hommes. Leur durée de vie est donc limitée. Dans la maison cérémonielle illustrée dans la photo 1 , certaines peintures de plafonds étaient tombées et les insectes y nichaient. Lorsque la maison est trop abîmée, les Kwoma retirent les tambours cérémoniels et certaines sculptures et laissent la maison à l'abandon. Lorsque qu'elle s'effondre, certaines poutres peuvent être récupérées. Si elles sont en bon état, elles peuvent être incorporées dans une nouvelle maison cérémonielle ou être vendues aux touristes ou à un collectionneur. La maison cérémonielle la plus récente a été commandée à Tongwinjamb pour la septième Triennale d'art contemporain d'Asie et du Pacifique. L'inauguration de cette maison a eu lieu le 5 août 2013. Elle est située à Tonga-3 au pied de la colline.

La construction d'une maison cérémonielle est supervisée par le propriétaire de la terre (apoko $m a)$ où est construite la maison, mais tous les membres du clan sont invités à participer à son érection, surtout s'ils comptent y tenir des activités. Lors de l'inauguration de la maison, les autres apoko ma du clan accroche au plafond un sac

7. L'igname est au centre d'un nombre important de rituels (Haudricourt, 1962), chez plusieurs peuples d'Océanie - par exemple chez les Kanak de Nouvelle-Calédonie (Leblic, 2002) - , et d'Afrique - par exemple chez les Fon du Bénin (Assogba, 2015).

8. Pour plus d'informations sur l'art kwoma, les lecteurs sont invités à consulter Bowden (2006). Il sera aussi important de spécifier qu'avec l'accès à la peinture moderne, de nouvelles couleurs ont fait leur entrée dans les ouvres kwoma, par exemple le vert et le bleu, comme on peut le voir dans la publication du musée du quai Branly-Jacques Chirac intitulé Rouge kwoma (Mélandri et Rovere, 2008). 
tressé chacun (kow ; tok pisin, bilum) rempli de la terre dont ils sont les propriétaires. La maison cérémonielle reçoit un nom issu d'un mythe connu. La maison visible sur la photo 1 porte le nom de Nokwasabawariweya. Il renvoie au mythe Teki selon lequel les chiens (asa) qui maltraitaient leurs femmes se les sont fait volées par les hommes. Les maisons cérémonielles sont situées au centre des villages ou au sommet d'une colline. Lorsqu'elles ne servent pas pour des cérémonies, elles offrent un lieu de réunion aux hommes (qui peuvent être accompagnés de leurs femmes si toutefois cellesci n'ont pas leurs règles ou si elles ne sont pas enceintes), afin de discuter des affaires du village. Le rituel de l'igname est composé de trois cérémonies: Yena, Mija et Nowkwi. Ces trois cérémonies font partie d'un cycle initiatique réservé aux hommes. Les meneurs de cérémonie sont des harapa ma "grands hommes", reconnus dans leur clan pour leur connaissance des chants, de la mythologie et de la procédure à suivre pour accomplir le rituel. À l'âge de la puberté, les jeunes hommes sont initiés au rituel de l'igname. Ils doivent d'abord choisir entre deux cérémonies distinctes, Yena, le rite lié à la récolte de l'igname, et Mija, le rite lié à la croissance de l'igname. Cette décision fera d'eux des Yenama "hommes Yena" ou Mijama "hommes Mija». Un homme conserve son appartenance cérémonielle toute sa vie. Toutefois, s'il acquiert assez de prestige dans l'accomplissement d'un rituel, Yena ou Mija, on lui permet de participer à l'autre rituel. Ainsi, un Yenama sera invité à participer à la cérémonie Mija et un Mijama à la cérémonie Yena. Ces hommes acquièrent davantage de prestige auprès des esprits puissants du clan, ceux à qui on attribue la responsabilité de la fertilité des jardins d'ignames. La troisième et dernière cérémonie, Nowkwi, symbolise la fertilité perpétuelle des jardins d'ignames. Elle vise à satisfaire les ancêtres pour obtenir dans le futur de bonnes récoltes. Traditionnellement, seuls ceux qui avaient tué un homme pouvaient devenir Nowkwima " hommes Nowkwi " (Whiting, 1941). Aujourd'hui, il suffit qu'un initié aux rituels Mija ou Yena ait acquis beaucoup de prestige dans son clan pour être introduit à la cérémonie Nowkwi. Ainsi, les initiés Nowkwi sont très âgés et peu nombreux. Jadis, seul un homme initié au rituel Nowkwi pouvait planter les ignames, car il apprenait lors de son initiation à solliciter la coopération des forces surnaturelles qui assuraient la fertilité du jardin. De nos jours, cette pratique a disparu, d'abord, parce que les hommes Nowkwi très peu nombreux sont souvent de constitution frêle et incapables d'accéder aux jardins d'ignames qui sont situés, généralement, à plus d'une heure de marche du village et à flanc de colline; ensuite, parce que la connaissance du rituel liée à la protection du jardin d'igname s'est perdue, ou du moins s'est altérée. Ainsi la cérémonie appelée kwar sukwiya n'a pas été célébrée depuis "le temps des bombes", c'est-à-dire depuis la Deuxième Guerre mondiale. Selon les récits de Whiting (1941), qui a assisté au rituel, et de Bowden (1977, 1983a), cette cérémonie enseignait les incantations et les chants indispensables à la plantation de l'igname et fournissait les savoirs sur les substances magiques qui étaient frottées sur les tubercules d'igname avant leur mise en terre. Cependant certaines pratiques héritées de la cérémonie kwar sukwiya subsistent toujours aujourd'hui, comme, par exemple, l'abstention de relations sexuelles pour plusieurs jours avant la plantation et jusqu'au début de la pousse des tubercules ou encore l'interdiction de manger certains aliments comme la viande de casoar et de sanglier par exemple.

Tel que rapporté par Whiting et Reed (1938) et Whiting (1941), le choix entre Yena et Mija était avant tout individuel. Les jeunes hommes faisaient l'objet de pression de la part de leurs pères, frères ainés et oncles pour choisir la cérémonie qui assurerait une représentation équilibrée des membres du clan dans chaque rituel. Cette pratique a évolué : Bowden (1977) rapporte par exemple qu'un père appartenant au rituel Yena assignait son fils ainé à la même section cérémonielle que lui, son second fils à la section Mija, son troisième à la section Yena, et ainsi de suite. Il est intéressant de noter que, d'après mes propres observations à Tongwinjamb quelques trente ans plus tard, l'assignation rituelle des hommes du clan est devenue héréditaire. Les fils d'un homme Mija sont assignés d'office à la section Mija et les fils d'un homme Yena à la section Yena. Plusieurs hommes s'affirmant Yena ou Mija ont même témoigné n'avoir jamais participé à une cérémonie de l'igname. Ils ont confirmé que le dernier rituel de l'igname avait eu lieu alors qu'ils étaient encore au lycée à Ambunti, ce qui, selon mes estimations, se situe au début des années 1990.

Lors de ces trois cérémonies, les hommes disposent différentes formes de sculptures en bois peint qui représentent les différents esprits, Yena et Mija. Chaque cérémonie est associée à un style de sculptures. La photo 2 montre une sculpture Mija masək " tête Mija " et son propriétaire, Benjamin, un Mijama de Tonga-2. Les sculptures Yena étaient traditionnellement considérées comme tabous, interdites aux regards des femmes et des étrangers. Cependant, l'intérêt des musées a rendu ces statues plus accessibles. Il est désormais possible de voir des sculptures Yena dans plusieurs musées, et on peut voir des photographies de celles-ci à la suite d'une simple recherche sur Internet. Les sculptures rituelles de l'igname sont parmi les plus connues de la culture du Sepik (voir Bowden, 1977, 1983a).

Le rituel de l'igname, avec ses trois différentes cérémonies initiatiques, est le plus important rituel des Kwoma. Autrefois, les meneurs de cérémonies s'assuraient que chaque génération puisse participer au cycle rituel de l'igname. L'arrivée du christianisme a chamboulé cet ordre, puisque les croyances traditionnelles étaient découragées par 


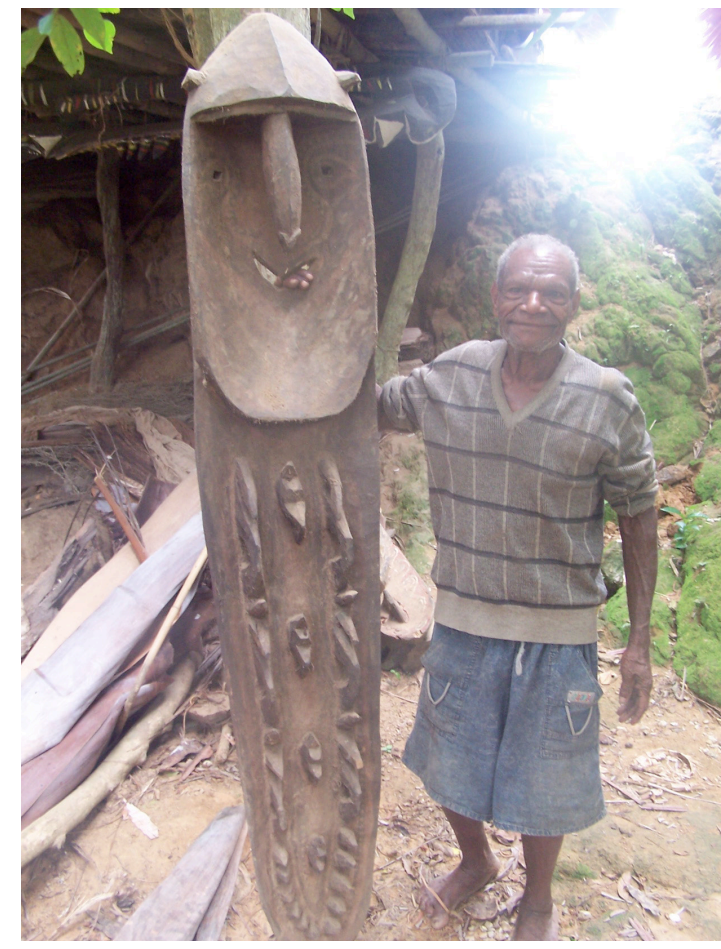

Рното 2. - Sculpture Mija et son propriétaire, Benjamin, un Mijama de Tonga-2, Tongwinjamb, 28 octobre 2008 (C) R. Lambert-Brétière)

les missionnaires. Une autre raison ayant entraîné la diminution du nombre de rituels est l'interdiction, par l'administration australienne, à la fin des années 1920, de la chasse aux têtes. Comme on l'a expliqué plus haut pour la cérémonie Nowkwi, la vie cérémonielle était organisée principalement par les guerriers. Ainsi, face à la disparition de ces acteurs, le système initiatique a progressivement décliné9. Comme l'explique Bowden (1983a), le rituel de l'igname a survécu malgré ces nombreux changements socioculturels. Ce fait est surprenant, puisque contrairement à d'autres sociétés voisines comme les Arapesh et les Abelam qui habitent les monts Torricelli au nord et où l'igname est à la fois la nourriture principale et source de rituels (voir par exemple Tuzin, 1972), les Kwoma ne consomment que très peu d'ignames (environ $5 \%$ de leur diète), leur nourriture principale étant le sagou et la patate douce. Une des raisons principales de ce maintien est que ce rituel détermine l'appartenance cérémonielle des hommes d'un clan et que cette appartenance joue un rôle primordial dans l'organisation sociale du village. Une autre raison est que le rituel de l'igname est un lieu d'apprentissage de la culture kwoma. Les garçons apprennent à jouer du tambour, à chan- ter et à danser afin de se préparer à l'initiation qui fera d'eux, à l'âge adulte, des Yenama ou des Mijama. Les hommes qui démontrent leurs talents lors de ces démonstrations artistiques gagnent en prestige dans la communauté. Ils peuvent espérer devenir des harapa ma "grands hommes" de leur clan. C'est dire qu'une grande partie du savoir culturel des Kwoma est transmis par le rituel de l'igname. La documentation existante sur le rituel de l'igname chez les Kwoma est avant tout axée sur son patrimoine matériel. Les sculptures des esprits Yena et Mija ont été étudiées et présentées dans divers musées et des expositions internationales (voir par exemple Mélandri et Rovere, 2008). Mais alors que ce patrimoine culturel matériel est relativement bien connu, sa contrepartie immatérielle l'est beaucoup moins. La section suivante porte sur une description de deux chants liés au rituel, sawo howkwa et magwiy howkwa.

\section{Les chants rituels}

Chacune des cérémonies du rituel de l'igname inclut des chants et des danses en l'honneur des esprits Yena, Mija ou Nowkwi. On y joue également du tambour (me; tok pisin, garamut), qui est un tronc d'arbre sculpté à une extrémité et pourvu d'une large cavité creusée en son milieu. Il est posé sur des pierres. Pour accompagner les chants, on le frappe à l'aide de maillets ${ }^{10}$. On y joue également des grandes flûtes de bambou, mais ces instruments étant sacrés, on ne m'a pas autorisée à les voir. J'ai choisi de décrire deux chants rituels qui accompagnent les cérémonies Yena et Mija ${ }^{11}$ On trouve d'abord les sawo howkwa, signifiant littéralement " le chant qui raconte». Ils sont des versions chantées d'une série de mythes appartenant aux clans kwoma (voir tableau 1). Le deuxième type de chants est dit magwiy howkwa "le chant du clan". Il porte sur les événements historiques et mythiques importants pour le clan. Mon choix s'est arrêté sur ces deux chants car ce sont ceux que j'ai pu entendre moi-même. Je tiens à préciser que je n'ai pas entendu ces chants dans le contexte d'un rituel de l'igname. Non seulement, ainsi que dit plus haut, le dernier rituel remonte à plusieurs années, mais il est interdit aux femmes et, ainsi, je n'aurais pas pu y assister.

\section{Sawo howkwa}

Les sawo howkwa sont des chants qui racontent et expliquent l'importance des figures mythiques

9. D'autres peuples du Sepik ont dû faire face à ces changements socioculturels, notamment les Iatmul, voisins des Kwoma (voir Bateson, 1932 ; Coiffier, 1992).

10. Voir Zemp et Kaufmann (1969) pour une description détaillée de l'utilisation des tambours chez les Kwoma.

11. La cérémonie Nowkwi fait intervenir un ensemble de chants différents de ceux entendus lors des deux autres cérémonies. Ces chants sont saba howkwa, le chant d'un père à sa fille qui se marie, et nowkwi howkwa, le chant de tous les clans, incluant des chants mythiques de clans non kwoma voisins. 
et autres esprits pour la division totémique. Ainsi, ces chants ne peuvent être produits que par des hommes membres du clan qui les possède. Dans leur version racontée, n'importe quel homme peut en faire la narration, mais, s'il n'en est pas le propriétaire, il débutera l'histoire en nommant le clan qui possède le mythe. Les femmes n'ont pas l'habitude de narrer les mythes traditionnels, néanmoins elles sont autorisées à le faire en privé. Ainsi, certains mythes de mon clan d'affiliation m'ont été racontés par des femmes, notamment le mythe lié à l'acquisition du feu (Atokob). Les sawo howkwa ne sont pas interdits aux femmes. J'ai pu en entendre quelques-uns chantés par Benjamin, un grand homme Mija de Tonga-2 (photo 2). Ces chants reposent sur des couplets. Lorsqu'ils ne sont pas chantés dans un cycle, ils sont très courts, en moyenne ils durent quarante secondes. Ils comprennent entre trois et dix phrases parfois répétées, comme le montre l'exemple 1 suivant. En théorie, tous les mythes peuvent être chantés, mais la version chantée differe de la version narrée en ce qu'elle présente les caractéristiques d'une variété de langue rituelle (Dubois, 2003). La compréhension du chant en (1) repose sur la maîtrise du langage rituel employé. On remarquera d'abord l'absence de verbes. Par exemple, la ligne (i), répétée à la ligne (iii), est une proposition relative sans verbe où le premier syntagme nominal est la tête et le deuxième modifie le premier dans une relation syntaxique illustrée en exemple 2 . La même proposition relative avec le verbe $t a$ " être " est montrée en (b) à titre illustratif.

La deuxième caractéristique notable de ce chant est la présence d'un vocabulaire spécifique aux mythes chantés. Le mot man aux lignes (vi) et (viii) est la forme courte du mot maji, prononcé "mangi " [manzi] en kwoma parlé. C'est un terme générique pour plusieurs types d'arbres dont l'écorce est utilisée pour tresser des sacs traditionnels, des filets de pêche, etc. L'arbre à pain est l'un de ces arbres. Un deuxième exemple est le mot sar aux mêmes lignes qui est la forme courte du mot saraga prononcé [saranga] et qui signifie " écorce ». Les plus jeunes qui étaient avec moi au moment où j'ai enregistré ce chant ne comprenaient pas ces mots, et Benjamin a dû en expliquer la signification. Un autre mot de vocabulaire qui différencie ce mythe chanté de sa version narrée est l'utilisation de l'adjectif egey " épineux " dans une relation métonymique avec wachi «arbre à pain " (Artocarpus camansi) pour référer au fruit à pain qui a une surface épineuse et qui est une source de nourriture chez les Kwoma. La façon courante de parler du fruit de l'arbre à pain est wachi sək où sək veut dire "fruit " ou " graine ".

Chaque ligne est chantée sur le même ton et se termine par un son [a] maintenu. De plus, entre les lignes (vi) et (vii), il y a une pause marquée par le son [a] qui diminue en intensité, comme le montre la forme d'ondes dans la Figure 1 obte-
(1)

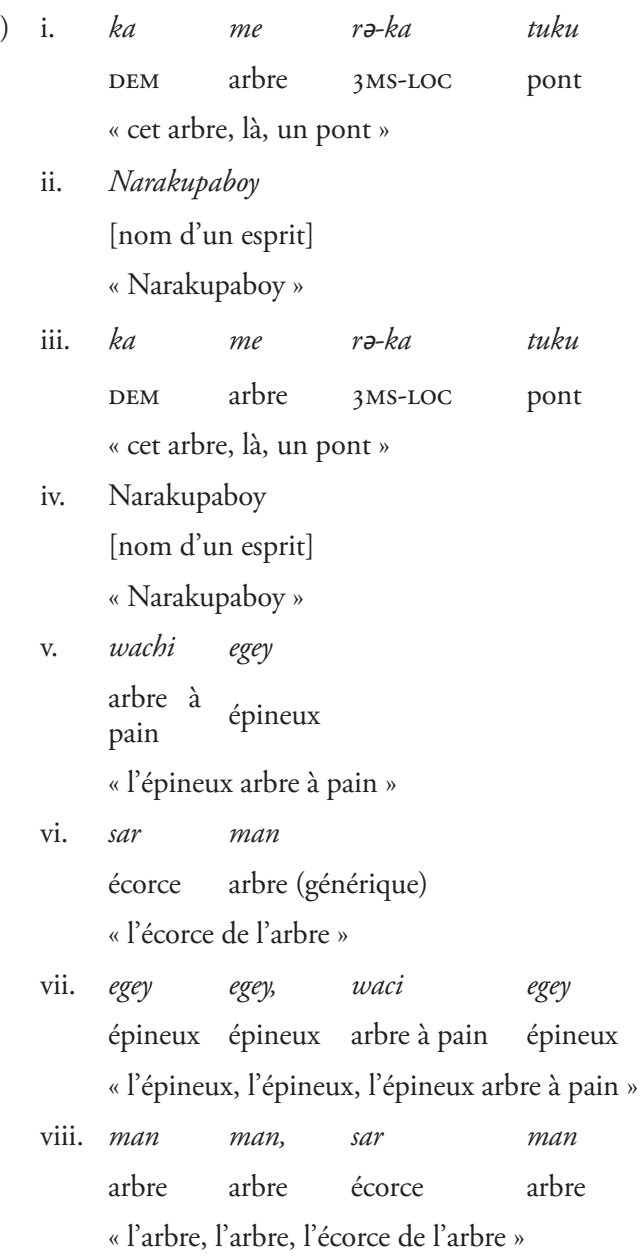

Exemple 1. - Chant Narakupaboy

Abréviations utilisées : 3: troisième personne; DEM : démonstratif; LOC : locatif ; MS : masculin singulier ; PERF : perfectif; SN : syntagme nominal; sv : syntagme verbal.

Exemple 2. - Proposition relative

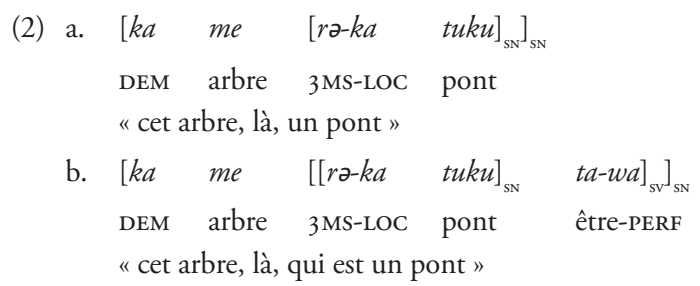

nue à l'aide du logiciel Pratt. Cet intermède dure presque quatre secondes et sépare deux strophes distinctes où la première - lignes (i) à (vi) contient l'information sur l'importance de Narakupaboy, et la deuxième - ligne (vii) et (viii) constitue une récapitulation des contributions de Narakupaboy à la culture Kwoma, c'est-à-dire le fruit à pain et son écorce.

Le chant se termine sur une série de sons [a] chantés et rythmés par Benjamin à l'aide une brindille 


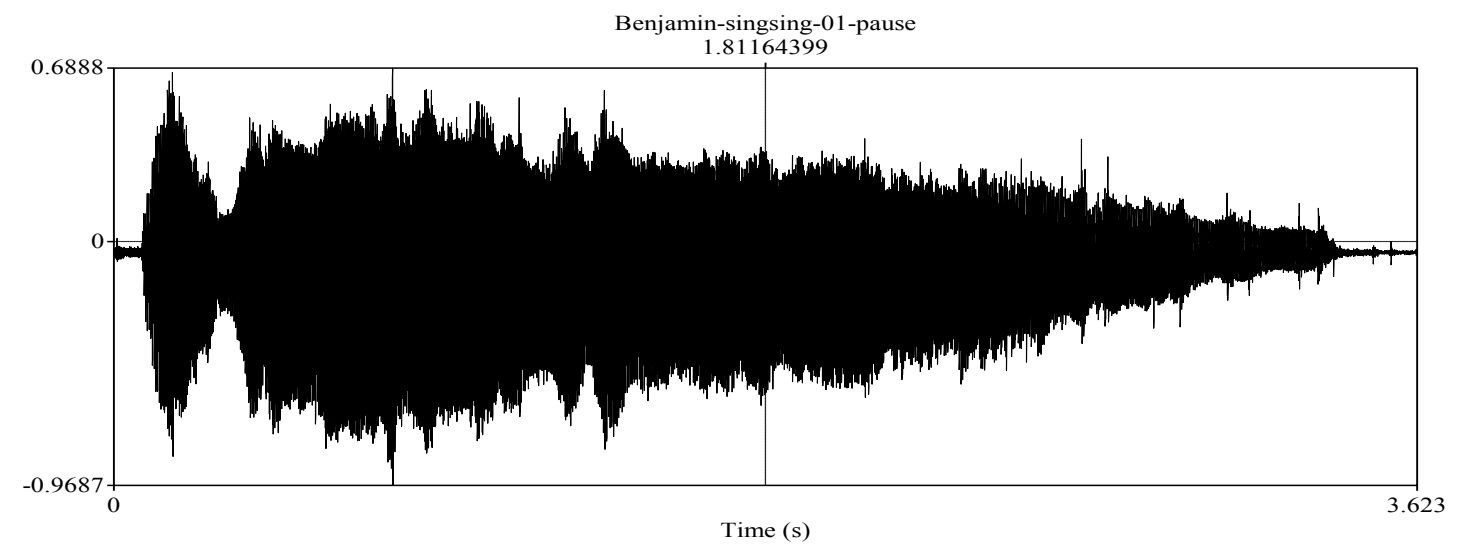

Figure 1. - Intermède entre les deux strophes du chant Narakupaboy

tapée sur une pierre. La signification de ce chant telle qu'elle nous a été donnée est la suivante :

"Cet arbre, là, qui est un pont

C'est Narakupaboy

Cet arbre, là, qui est un pont

C'est Narakupaboy

L'arbre à pain nous a donné son fruit

Larbre à pain nous a donné son écorce

Le fruit, le fruit, le fruit de l'arbre à pain

L'écorce, l'écorce, l'écorce de l'arbre à pain "

Les sawo howkwa sont chantés comme divertissement et lors des grandes manifestations publiques comme la construction d'une maison. Les sawo howkwa sont également les premiers chants entendus lors des cérémonies rituelles de l'igname. Suivant la description de Bowden (1983a : 71), il n'y a pas d'ordre précis dans lequel ces chants doivent être réalisés. Les meneurs de chants sélectionnent les couplets qu'ils connaissent le mieux ou pour lesquels ils ont un intérêt particulier.

\section{Magwiy howkwa}

Le deuxième type de chants rituels que je décrirai ici s'appelle magwiy howkwa "le chant du clan". Suivant l'estimation de Bowden (1983a: 113), il y aurait une quinzaine de cycles de chants claniques, chacun contenant entre cent et trois cents couplets distincts. Seuls les meneurs de cérémonies connaissent la majeure partie des chants. Ce cycle de chants est au cœur des cérémonies Yena et Mija : il est chanté pendant la plus grande partie du rituel. À plusieurs reprises lors de mon séjour en 2007 , on nous annonça la tenue d'un chant traditionnel du clan Hamikwa. Plus d'une fois, on nous avertit que le chant serait produit dans la soirée, mais en réalité l'événement n'avait pas lieu. L'attente dura plusieurs jours. Ce chant n'est pas connu de beaucoup de villageois. Comme dans l'exemple précédent, le magwiy howkwa est com- posé d'une langue kwoma ancienne partiellement incomprise par les jeunes générations.

Les restrictions culturelles entourant les interactions entre hommes et femmes à Tongwinjamb et en Papouasie Nouvelle-Guinée en général ont en quelque sorte déterminé mon niveau d'accès ou non à certain matériau culturel. Alors que j’ai pu entendre quelques chants du type sawo howkwa, je n'ai pas été invitée à la cérémonie où le $m a-$ gwiy howkwa a été entendu. En revanche, mon mari a assisté à la performance à l'intérieur de la maison cérémonielle. Celle-ci, pour la première fois de notre séjour, était remplie, ce qui dénotait l'importance de l'événement. Quelques hommes étaient assis sur les grands tambours, mais la plupart se tenaient debout. Beaucoup d'hommes étaient venus avec leur tambour portatif (wokw). Ce sont des morceaux de troncs d'environ quarante centimètres de diamètre sur soixante-dix centimètres de longueur qui sont sculptés en forme de sablier. Leur centre est entièrement creusé. Une anse est ménagée au centre du tambour afin de le tenir. Une peau, souvent de lézard, est tendue sur l'une des extrémités. Les alentours de la maison cérémonielle étaient bondés de groupes de femmes et d'enfants prêts à écouter le chant de l'extérieur. Une table était installée proche de l'entrée et une bonne demi-douzaine de lecteurs-enregistreurs de cassettes fut posée sur la table par des hommes de quinze à trente ans. Suivant mon expérience dans le village, il n'est pas coutume d'enregistrer les chants. La particularité du chant ce soir-là était qu'il serait l'un des plus complets. Selon les dires de plusieurs personnes, cette version longue n'avait pas été exécutée depuis plusieurs années.

Dès la nuit tombée, un jeune homme que nous n'avions pas rencontré auparavant prit la parole. C'était le petit-fils du harapa ma de la maison cérémonielle où se produisait le chant. Il était la cause de la remise incessante de la performance: on attendait sa venue d'Ambunti. Il était les yeux de son grand-père aveugle et devait prendre sa place 
comme maitre de cérémonie. Il expliqua d'abord que le chant appartient aux Hamikwa et que, selon la tradition, il ne peut être chanté qu'une fois par an. Seuls les Kwoma et les membres de leur famille ont le droit de l'entendre. Comme beaucoup de gens ont regardé mon mari à ce momentlà, le maître de cérémonie a réitéré l'autorisation que nous avions d'écouter le chant à cause de ma relation avec ma consultante principale, non seulement mon premier contact chez les Kwoma, mais aussi ma grande sœur dans le clan (mowey). Il a répété à plusieurs reprises que bien que l'enregistrement fut autorisé, il était interdit de le faire écouter à des non-Kwoma et que je ne pouvais pas utiliser les paroles du chant dans mes publications. Il a ensuite précisé que, par extension, nos parents avaient le droit d'écouter le chant, si nous nous assurions que personne d'autre ne pouvait l'entendre. Le maître de cérémonie termina son intervention en désignant la dizaine d'hommes aînés qui allaient entamer le chant.

Le chant dura environ une heure. Il fut divisé en couplets qui duraient entre cinq et dix minutes, comprenant les refrains repris en cœur par la majorité des hommes et accompagnés par les battements des tambours. Deux anciens du village, âgés d'une soixantaine d'années, ont chanté la majorité des couplets. Les chanteurs se relayaient régulièrement après un, deux ou trois couplets. Quelques couplets furent chantés à deux ou trois voix, plusieurs hommes voulant chanter le même couplet. Après une quinzaine de minutes continues, le chant était souvent interrompu à la fin du refrain, certains chanteurs ne connaissant pas les paroles. Les plus jeunes nous expliquèrent qu'il est difficile de mémoriser les paroles car, outre le fait qu'ils n'entendent pas souvent ce chant, ils ne comprennent plus certains mots qui relèvent de formes archaïques.

Entre le chant et les pauses, la performance a duré deux heures. L'importance de magwiy howkwa pour le clan s'est reflétée dans le nombre d'hommes présents dans la maison cérémonielle, le nombre de femmes et d'enfants à l'extérieur, la quantité de feux illuminant l'intérieur de la maison et le battement des nombreux tambours. Alors que les sawo howkwa sont chantés lors de célébrations publiques, les magwiy howkwa ne sont chantés au maximum qu'une fois par an à l'intérieur d'une maison cérémonielle. Ce chant étant rarement entendu, l'événement a entraîné la mobilisation de tous les villageois qui connaissaient tout ou une partie du chant, et ceci, quel que fut leur totem. Il a fallu attendre que la réunion de ces villageois soit possible pour que le magwiy howkwa soit chanté, ce qui a pris plusieurs jours. La promesse de l'enregistrement et la présence des chanteurs expliquent qu'un grand nombre de familles se soit déplacé ce soir-là, remplissant la grande maison traditionnelle et ses alentours.

\section{Langue et savoir culturel en danger}

Malgré les nombreux bouleversements de la vie des Kwoma, le rituel de l'igname demeure encore aujourd'hui leur rituel le plus important. Le rôle de ce rituel dépasse largement celui de la culture et de la récolte de l'igname. Il fait partie intégrante du processus de continuité culturelle, c'est-à-dire qu'il est au cour du lien intergénérationnel maintenu à travers la transmission des traditions aux générations suivantes. Cette continuité n'exclut pas la transformation du patrimoine culturel, mais assure la cohésion sociale et culturelle au sein de la communauté. Une grande quantité des savoirs historiques, culturels et spirituels sont transmis par les chants rituels sawo howkwa et magwiy howkwa interprétés lors des cérémonies Yena et Mija. Les chants rituels renseignent sur les savoirs culturels d'importance pour les Kwoma. Ils conceptualisent l'identité des Kwoma en l'ancrant dans des idéologies historiques: ils sont une source de savoir sur l'histoire et sur les valeurs et normes socioculturelles attendues dans cette communauté. Or, la transmission de cette tradition linguistique est elle-même menacée par le fait que les cérémonies du rituel de l'igname n’ont pas été célébrées depuis la fin des années 1980. Comme les chants constituent une grande partie des célébrations, la non-performance du rituel entraîne avec elle une perte de la connaissance de ces chants. La langue rituelle qui compose les sawo howkwa et les magwiy howkwa n'est plus connue que par les quelques harapa ma restants. Comme nous en avons été les témoins mon mari et moi, le kwoma ancien employé dans les chants est difficile à comprendre pour les villageois de moins de cinquante ans. Le magwiy howkwa a été chanté, comme indiqué plus haut, dans une version particulièrement complète en raison de la présence des connaisseurs du chant. Trois harapa ma étaient assis à l'entrée de la maison cérémonielle pour toute la durée du chant. Ils ont souvent été consultés pour des clarifications sur les paroles ou pour l'ajout de couplets. Ma grande sour Betty est l'une des femmes qui a assisté à la cérémonie de l'extérieur de la maison cérémonielle. Les femmes n'étant pas autorisées à entamer un chant, personne ne l'a interrogée, mais à quelques reprises, le silence s'est établi dans la maison afin de l'entendre lancer les paroles que les chanteurs allaient répéter dans les couplets.

Le rituel de l'igname est l'un des mieux connus des rituels kwoma, mais il est grandement menacé par la perte du savoir linguistique en permettant sa réalisation. La transformation de la vie rituelle des Kwoma a laissé derrière elle peu d'hommes capables de réaliser les trois cérémonies, Yena, Mija et Nowkwi, composant ce rituel. La préservation du rituel de l'igname est indissociable du processus vivant de sa transmission par la langue, et ainsi, la sauvegarde du 
patrimoine culturel ne peut se faire aux dépens de la sauvegarde du patrimoine immatériel.

\section{BIBLIOGRAPHIE}

Aikhenvald Alexandra Y. et Tonya Stebbins, 2007. Languages of Papua New Guinea, in O. Miyaoka, O. Sakiyama et M. Krauss (eds), Vanishing Languages of the Pacific, Oxford, Oxford University Press, pp. 239-66.

ARpin Roland, 2001. La langue, notre premier patrimoine, Québec français 121, pp. 39-41.

Assogba C. Raymond, 2015. Rituels de l'igname, altérité et contracculturation en Afrique noire, Sarrebruck, Presses académiques francophones.

Bateson Gregory, 1932. Social Structure of the Iatmul People of the Sepik River, Oceania 2, pp. 246-290.

Bornand Sandra et Cécile Leguy, 2013. Anthropologie des pratiques langagières, Paris, Armand Colin.

Bowden Ross, 1977. The Kwoma: A Study of Terminology and Marriage Alliance in a Sepik River Society, Thèse de doctorat, Monash University.

—, 1983a. Yena. Art and Ceremony in a Sepik Society, Oxford, Pitt Rivers Museum.

—, 1983b. Kwoma Terminology and Marriage Alliance: The 'Omaha' Problem Revisited, Man 18, pp. 745-765.

—, 1997. A Dictionary of Kwoma. A Papuan Language of North-East New Guinea, Canberra, Pacific Linguistics.

—, 2006. Creative Spirits. Bark Painting in the Washkuk Hills of North New Guinea, Melbourne, Oceania Arts.

CoIffier Christian, 1992. Rituels et identité culturelle iatmul (Vallée du Sepik - Papouasie Nouvelle-Guinée), Bulletin de l'École française d'Extrême-Orient 79, pp. 131-148.

Crystal David, 2000 ( $2^{\text {nd }}$ ed.). Language Death. Cambridge, Cambridge University Press.

DuBors John, 2003. Ritual Language, in W. J. Frawley (ed.), International Encyclopedia of Linguistics, Oxford, Oxford University Press.

Duranti Alessandro, 1997. Linguistic Anthropology, Cambridge, Cambridge University Press.

Foley William A., 1986. The Papuan languages of New Guinea, Cambridge, Cambridge University Press.

—, 1998. Toward understanding Papuan languages, in J. Miedema, C. Odé et R.A.C. Dam (eds), Perspectives on the Bird's Head of Irian Jaya, Indonesia, Amsterdam, Rodopi BV, pp. 503-518.
_, 2000. The Languages of New Guinea, Annual Review of Anthropology 29, pp. 357-404.

Grattan David, 2006. Le patrimoine immatériel et la conservation - la part entre l'usage et la préservation, Bulletin de l'Institut canadien de conservation 37(http://www.cci-icc.gc.ca/cciicc/about-apropos/nb/nb37/usage-fra.aspx).

Harrison K. David, 2007. When Languages Die. The Extinction of the World's Languages and the Erosion of Human Knowledge, Oxford, Oxford University Press.

Haudricourt André-Georges, 1964. Nature et culture dans la civilisation de l'igname : l'origine des clones et des clans, L'Homme 4, pp. 93-104.

Le Tollec François, 2013. De la tradition orale à la préservation de l'expression: Transmission ou interprétation d'un langage, Synergies Mexique 3, pp. 133-142.

LeBlic Isabelle, 2002. Ignames, interdits et ancêtres en Nouvelle-Calédonie, Journal de la Société des Océanistes 114-115, pp. 115-127 (https://journals.openedition.org/jso/1439).

Martinet André, 2005. Eléments de linguistique générale, Paris, Armand Colin, $4^{\mathrm{e}}$ édition.

Maynard Smith John and Eörs Szathmáry, 1995. The Major Transitions in Evolution, Oxford, Oxford University Press.

MéLANDri Magali et Maxime Rovere, 2008. Rouge kwoma. Peintures mythiques de NouvelleGuinée, Paris, musée du quai Branly/RmN.

Simons Gary F. et Charles D. Fennig (eds), 2017. Ethnologue: Languages of the World, Twentieth edition, Dallas, sIL International.

Tuzin Donald F., 1972. Yam Symbolism in the Sepik, Southwestern Journal of Anthropology 28, pp. 230-254.

Von Humboldt William, 1859 [1836]. De l'origine des formes grammaticales et de leur influence sur le développement des idées, traduit par Alfred Tonnellé, Paris, A. Franck.

Whiting John W. M., 1941. Becoming Kwoma: Teaching and Learning in a New Guinea Tribe, New Haven, Yale University Press.

Whiting John W. M. and Stephen W. Reed, 1938. Kwoma Culture: Report on Field Work in the Mandated Territory of New Guinea, Oceania 9, pp. 170-216.

Zemp Hugo et Christian Kaufmann, 1969. Pour une transcription automatique des "langages tambourinés " mélanésiens : un exemple kwoma, Nouvelle-Guinée, L'Homme 9, pp. 38-88. 
